\title{
REDUCED COMPLEXITY IN-PHASE/QUADRATURE-PHASE TURBO EQUALISATION USING ITERATIVE CHANNEL ESTIMATION
}

\author{
B. L. Yeap, C. H. Wong and L. Hanzo \\ Dept. of Electr. and Comp. Sc., Univ. of Southampton, SO17 1BJ, UK. \\ Tel: +44-2380-593 125, Fax: +44-2380-593045 \\ Email: 1h@ecs.soton.ac.uk \\ http://www-mobile.ecs.soton.ac.uk
}

\begin{abstract}
A novel reduced complexity trellisbased equaliser, referred to as the In-phase/Quadrature-phase Equaliser (I/Q-EQ), is proposed. The $I / Q-E Q$ is employed in the context of turbo equalisation (TEQ-IQ) and with the aid of iterative channel estimation. The performance of the TEQ-IQ is characterized in a noise limited environment over an equally-weighted, symbolspaced three-path Rayleigh fading channel. The TEQ-IQ achieved the same performance as the conventional turbo equaliser, while achieving a complexity reduction by a factor of 1.25 and 7.17 for 4-QAM and 16-QAM, respectively.
\end{abstract}

\section{INTRODUCTION}

In a communications system, the received signal is degraded by Inter Symbol Interference (ISI) introduced by the channel. The effects of ISI can be mitigated by employing equalisation and the associated Bit Error Rate (BER) can be further reduced by using error correction schemes. However, when performing the equalisation and channel decoding independently, we cannot compensate for the performance loss due to the ISI completely, even when soft decisions are passed from the equaliser to the channel decoder. Instead, by performing the channel equalisation and decoding jointly, as in the iterative turbo equalisation scheme proposed by Douillard et al. [1], the residual ISI can be substantially mitigated. Gertsman and Lodge [2] then showed that the iterative process of turbo equalisation can be exploited to compensate for the performance degradations due to imperfect channel estimation. Knickenberg et al. [3] subsequently proposed a non-iterative joint equalisation and decoding technique based on a supertrellis structure. This technique yielded an optimum performance, but it was restricted to incorporating simple interleavers due to the high complexity incurred in conjunction with interleavers.

Due to complexity reasons, early turbo equalisation investigations using the conventional trellis-based equaliser (CT-EQ) were constrained to applying Binary Pha- se Shift Keying (BPSK) and Quadrature Phase Shift Keying (QPSK) modulation schemes [2]. Furthermore, only a limited Channel Impulse Response (CIR) duration could be equalised since the computational complexity incurred by the CT-EQ is dependent on both the maximum CIR duration and on the modulation mode utilised. Hence, turbo equalisation research has been focused on developing reduced complexity equalisers, such as the low-complexity linear equaliser proposed by Glavieux et al. [4] and the Radial Basis Function RBF equaliser of Yee et al. [5].

Motivated by these trends, we propose a novel reduced complexity trellis-based channel equaliser, referred to as the In-phase/Quadrature-phase Equaliser (I/Q-EQ), in the context of turbo equalisation invoking iterative channel estimation [6] for high throughput M-level Quadrature Amplitude Modulation (M-QAM). The performance of this reduced-complexity I/Q turbo equaliser (TEQ-IQ) is compared to that of the conventional turbo equaliser (TEQ-CT) benefiting from perfect CIR. The basic principle of the reduced complexity equaliser is based on equalising the in-phase (I) and quadrature-phase (Q) component of the transmitted signal independently. Therefore, the number of states for the in-phase and quadrature-phase trellisbased equaliser is reduced, when compared to the aforementioned CT-EQ. This issue will be made more explicit during our forthcoming discourse.

\section{PRINCIPLE OF I/Q EQUALISATION}

When the modulated signal $s(t)$ is transmitted over the channel characterised by the CIR $h(t)$ and further corrupted by the zero-mean complex white Gaussian noise $n(t)$ having a variance of $\sigma^{2}=N_{o} / 2$, where $N_{o}$ is the single-sided noise power spectral density, the received signal $r(t)$ becomes:

$$
\begin{aligned}
& r(t)=s(t) * h(t)+n(t) \\
& \quad=\left[s_{I}(t)+j s_{Q}(t)\right] *\left[h_{I}(t)+j h_{Q}(t)\right]+n_{I}(t)+j n_{Q}(t) \\
& \quad=r_{I}(t)+j r_{Q}(t),
\end{aligned}
$$




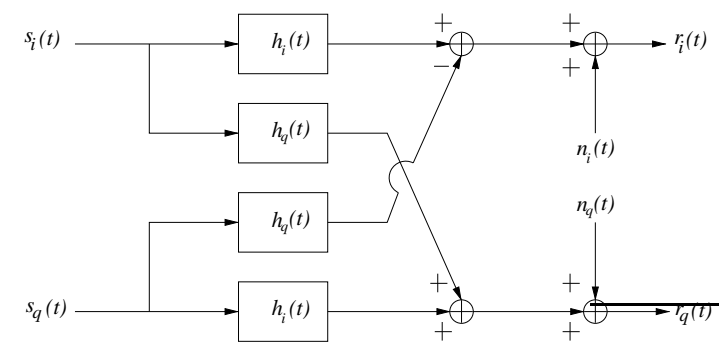

Figure 1: Model of the complex channel. After transmission over the complex channel $h(t)$, the received signal $r(t)$ becomes dependent on the in-phase component $s_{I}(t)$ and quadrature-phase component $s_{Q}(t)$ of the transmitted signal, as expressed in Equations 1 and 2 .

where

$$
\begin{aligned}
r_{I}(t) & =s_{I}(t) * h_{I}(t)-s_{Q}(t) * h_{Q}(t)+n_{I}(t) \\
r_{Q}(t) & =s_{I}(t) * h_{Q}(t)+s_{Q}(t) * h_{I}(t)+n_{Q}(t),
\end{aligned}
$$

since the CIR $h(t)$ is complex and therefore consists of the I component $h_{I}(t)$ and Q component $h_{Q}(t)$. Similarly, $s_{I}(t)$ and $s_{Q}(t)$ are the $\mathrm{I}$ and $\mathrm{Q}$ components of the transmitted signal $s(t)$, as illustrated in Figure 1, while $n_{I}(t)$ and $n_{Q}(t)$ are the quadrature components of the Gaussian noise $n(t)$. As shown in Equation 2, the received I/Q signals, namely $r_{I}(t)$ and $r_{Q}(t)$, become dependent on $s_{I}(t)$ and $s_{Q}(t)$ after transmission over the complex channel. We refer to the inter-dependency between $s_{I}(t)$ and $s_{Q}(t)$ in the received quadrature signals $r_{I}(t)$ and $r_{Q}(t)$ as cross-coupling. This crosscoupling of the transmitted signal's quadrature components requires the receiver to consider an increased number of signal combinations, hence necessitating a high number of equaliser trellis states. However, we can reduce the number of states to be considered significantly, when the cross-coupling is removed such that the quadrature components of the decoupled channel output $r^{\prime}(t)$ are only dependent on $s_{I}(t)$ or $s_{Q}(t)$, as illustrated in Figure 2 and augmented below:

$$
\begin{aligned}
r_{I}^{\prime}(t) & =s_{I}(t) * h(t)+n_{I}(t) \\
& =s_{I}(t) * h_{I}(t)+j\left[s_{I}(t) * h_{Q}(t)\right]+n(t) \\
r_{Q}^{\prime}(t) & =-s_{Q}(t) * h(t)+n_{Q}(t) \\
& =-\left(s_{Q}(t) * h_{I}(t)+j\left[s_{Q}(t) * h_{Q}(t)\right]\right)+n(t) .
\end{aligned}
$$

This is achieved by generating the estimates $\hat{s}_{I}(t)$ and $\hat{s}_{Q}(t)$ of the transmitted signal [4] - using the reliability information output by the decoder - and, as shown in Figure 2, by cancelling the cross-coupling effects of the transmitted quadrature signals from both the I and $\mathrm{Q}$ received signal components, namely from $r^{\prime}{ }_{I}(t)$ and $r_{Q}^{\prime}(t)$, respectively. In the ideal scenario, where perfect signal regeneration is possible, the cross-coupling inherent in the received signal can be successfully removed. No doubt that there will be errors introduced in

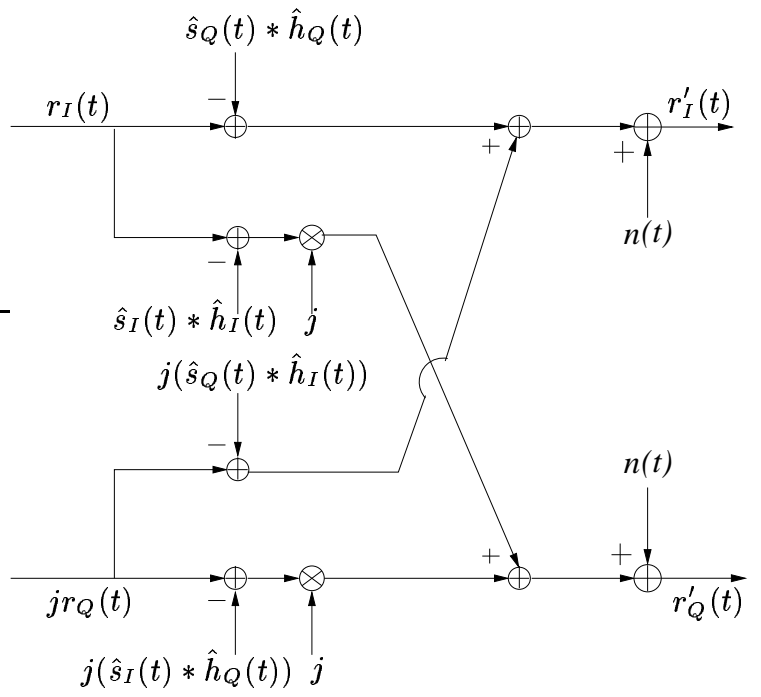

Figure 2: Removing the dependency of $r_{I}(t)$ and $r_{Q}(t)$ on the quadrature components of the transmitted signals, namely $s_{I}(t)$ and $s_{Q}(t)$, to give $r_{I}^{\prime}(t)$ and $r_{Q}^{\prime}(t)$, respectively. In this figure, it is assumed that the CIR estimation is perfect, i.e. $\hat{h}_{I}(t)=h_{I}(t)$ and $\hat{h}_{Q}(t)=h_{Q}(t)$ and that the transmitted signals are known, giving $\hat{s}_{I}(t)=s_{I}(t)$ and $\hat{s}_{Q}(t)=s_{Q}(t)$. In this case, perfect decoupling is achieved. However, in practice these estimates have to be generated at the receiver.

the decoupling operation, when inaccurate symbol estimates are generated from the channel-impaired lowconfidence reliability values. However, as seen in the simulation results of Section VII, the imperfect decoupling effects are compensated through successive turbo equalization iterations and the performance approaches that of the turbo equalizer utilising the conventional trellis-based equalizer.

After the decoupling operation, the modified complex channel outputs, namely $r_{I}^{\prime}(t)$ and $r_{Q}^{\prime}(t)$, respectively, can be viewed as the result of convolving both quadrature components independently with the complex CIR on each quadrature arm. Consequently, we can equalise $s_{I}(t)$ and $s_{Q}(t)$ independently, hence reducing the number of states in the trellis significantly. Again, note that in Equations 3 we have assumed that perfect signal regeneration and perfect decoupling is achieved at the receiver, in order to highlight the underlying principle of the reduced complexity equaliser. The schematic of the reduced complexity equaliser using trellis-based equalisers is illustrated in Figure 5.

\section{SYSTEM OVERVIEW}

The schematic of the entire system is shown in Figure 3 , where the transmitted source bits are convolutionally encoded, interleaved and mapped to a modulation symbol. The encoder utilised a $\frac{1}{2}$-rate Recursive Sys- 


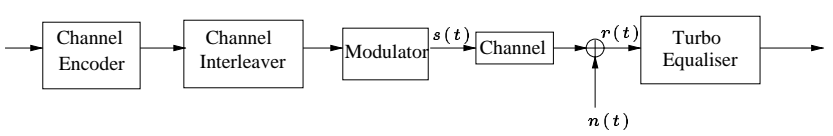

Figure 3: A coded M-QAM system employing a turbo equaliser at the receiver.

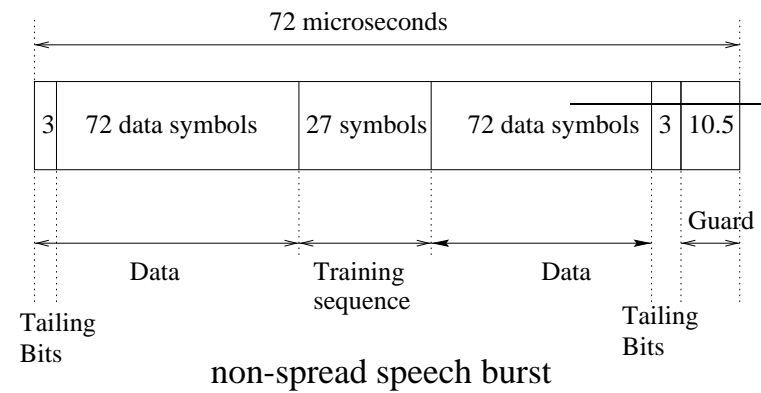

Figure 4: Transmission burst structure of the FMA1 non-spread speech burst of the FRAMES proposal [7].

tematic Convolutional (RSC) code having a constraint length of $K=5$ and octal generator polynomials of $G_{0}=35$ and $G_{1}=23$. In our investigations, the transmission delay of the 4-QAM, 16-QAM and 64-QAM systems [8] was limited to approximately $30 \mathrm{~ms}$. This corresponds to 3456 symbols at a symbol rate of 13.9 Kbauds and hence 6912-bit, 13824-bit and 20736-bit random channel interleavers were utilised for 4-QAM, 16-QAM and 64-QAM, respectively. The transmission burst structure used in this system is the FMA1 non-spread speech burst specified by the Pan-European FRAMES proposal [7], which is shown in Figure 4. A three-path, symbol-spaced fading CIR of equal weights was used, which can be expressed as:

$$
h(t)=0.577+0.577 z^{-1}+0.577 z^{-2},
$$

where the Rayleigh fading statistics obeyed a normalised Doppler frequency of $3.3615 \times 10^{-5}$. In our investigations the fading magnitude and phase was kept constant for the duration of a transmission burst, a condition which we refer to as employing transmission burstinvariant fading.

Figure 5 illustrates the schematic of the turbo equaliser utilising two reduced complexity I/Q-EQs. Since the Log-MAP algorithm [9] is employed in the I/QEQ and in the channel decoder blocks, the soft decisions generated are in the form of Log-Likelihood Ratios (LLRs). Here, we expressed the LLR of the equaliser and decoder using vector notations, according to the approach of [2], but using different specific notations. The superscript denotes the nature of the LLR, namely ' $c$ ' is used for the composite a posteriori [10] information, ' $i$ ' [10] for the combined channel and extrinsic information and ' $e$ ' [10] for the extrinsic information. Furthermore, the subscripts in Figure 5 are used to represent the iteration index, while the argument within the brackets ( ) indicates the index of

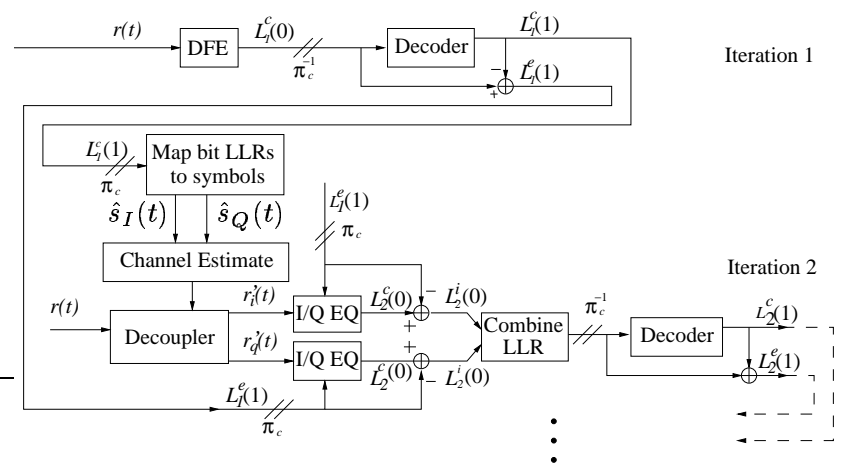

Figure 5: Schematic of the turbo equaliser employing a DFE and a SISO channel decoder in the first turbo equalisation iteration. In subsequent iterations, two I/Q-EQs and one SISO channel decoder is employed. The notation $\pi_{c}$ represents a channel interleaver, while $\pi_{c}^{-1}$ is used to denote a channel deinterleaver.

the receiver stage, where the equalisers are denoted as stage 0 , while the channel decoder as stage 1 .

At the receiver, the CIR was estimated using the Least Mean Square (LMS) algorithm [11] and the training symbols of the transmission burst are shown in Figure 4. The initial step-size of the LMS algorithm was set to 0.05. This initial CIR estimate was then utilised during the first turbo equalisation iteration by the conventional Decision Feedback Equaliser (DFE), as seen in Figure 5. The reason for employing the DFE in the first turbo equalisation iteration was to provide soft decisions in the form of the LLR $L_{1}^{c}(0)$ to the channel decoder. Invoking the DFE at the first iteration constituted a low-complexity approach to providing initial estimates of the transmitted symbols, as compared to the more complex CT-EQ. Subsequently, the Soft-In/SoftOut (SISO) channel decoder of Figure 5 generates the a posteriori LLR $L_{1}^{c}(1)$ and then the extrinsic information of the encoded bits, namely $L_{1}^{e}(1)$ is extracted.

In the next iteration, the a posteriori LLR $L_{1}^{c}(1)$ is used to regenerate estimates of the I and Q components of the transmitted signal, namely $\hat{s}_{I}(t)$ and $\hat{s}_{Q}(t)$, as seen in the 'MAP bit LLRs to symbols' block of Figure 5. The a posteriori information was transformed from the log domain to modulated symbols using the approach employed in [4]. At this stage, the CIR is re-estimated and refined with a smaller step-size of 0.01 using the regenerated symbols of the entire transmission burst. The estimated transmitted quadrature components $\hat{s}_{I}(t)$ and $\hat{s}_{Q}(t)$ are then convolved with the estimate of the CIR $h(t)$. At the decoupler block of Figure 5, the resultant signal is used to remove the cross-coupling effect - seen in Equation 2 - according to Equation 3 from both quadrature components of the transmitted signal, yielding $r^{\prime}{ }_{I}(t)$ and $r_{Q}^{\prime}(t)$.

After the decoupling operation, $r^{\prime}{ }_{I}(t)$ and $r_{Q}^{\prime}(t)$ are passed to the I/Q-EQ in the schematic of Figure 5. In 
addition to these received quadrature signals, the I/QEQ also processes the a priori information received which is constituted by the extrinsic LLRs $L_{1}^{e}(1)$ from the previous iteration - and generates the a posteriori information $L_{2}^{c}(0)$. Subsequently, the combined channel and extrinsic information $L_{2}^{i}(0)$ is extracted from both I/Q-EQs in Figure 5 and combined, before being passed to the Log-MAP channel decoder. As in the first turbo equalisation iteration, the a posteriori and extrinsic information of the encoded bits, namely $L_{2}^{c}(1)$ and $L_{2}^{e}(1)$, respectively, are evaluated. The following turbo equalisation iterations also obey the same sequence of operations, until the iteration termination criterion is met.

\section{SYSTEM COMPLEXITY}

The complexity $\Gamma$ of the CT-EQ and that of the I/QEQ for square-constellation M-QAM systems is expressed here for the sake of simplicity in terms of the number of trellis transitions per information bit. For the CT-EQ, the complexity associated with equalising M-QAM signals transmitted over a complex channel having a delay spread of $\tau_{d}$ symbols is:

$$
\begin{aligned}
\Gamma(\mathrm{CT}-\mathrm{EQ}) & =\frac{\text { Number of states } \cdot \text { Number of transitions }}{R} \\
& =\frac{\mathrm{M}^{\tau_{d}} \cdot \mathrm{M}}{R}=\frac{\mathrm{M}^{\tau_{d}+1}}{R},
\end{aligned}
$$

where $R$ is the code rate. For one I/Q-EQ trellis stage, we have:

$$
\begin{aligned}
\Gamma(\mathrm{I} / \mathrm{Q}-\mathrm{EQ}) & =\frac{\text { Number of states } \cdot \text { Number of transitions }}{R} \\
& =\frac{\sqrt{\mathrm{M}}^{\tau_{d}} \cdot \sqrt{\mathrm{M}}}{R}=\frac{\sqrt{\mathrm{M}}^{\tau_{d}+1}}{R} .
\end{aligned}
$$

However, in order to evaluate the total complexity $\Gamma$ of the turbo equaliser, which performs iterative equalisation and decoding jointly, we also have to consider the number of iterations $I_{t}$, which is a multiplicative complexity factor. The corresponding expressions for the total receiver complexity can therefore be formulated as:

$$
\begin{gathered}
\Gamma(\mathrm{TEQ}-\mathrm{CT})=I_{t}(\mathrm{TEQ}-\mathrm{CT}) \times\left(\frac{\mathrm{M}^{\tau_{d}+1}}{R}+2^{K}\right) \\
\Gamma(\mathrm{TEQ}-\mathrm{IQ})=I_{t}(\mathrm{TEQ}-\mathrm{IQ}) \times\left(\frac{2 \sqrt{\mathrm{M}}^{\tau_{d}+1}}{R}+2^{K}\right),
\end{gathered}
$$

where $2^{K}$ is the number of transitions considered at each decoder trellis interval and $K$ is the constraint length of the convolutional code. A factor ' 2 ' is included in Equation 7, since two I/Q-EQs are employed in the TEQ-IQ receiver. Note that in order to arrive at a relatively simple TEQ-IQ complexity expression, the DFE has been assumed to have the same complexity as that of the I/Q-EQ.

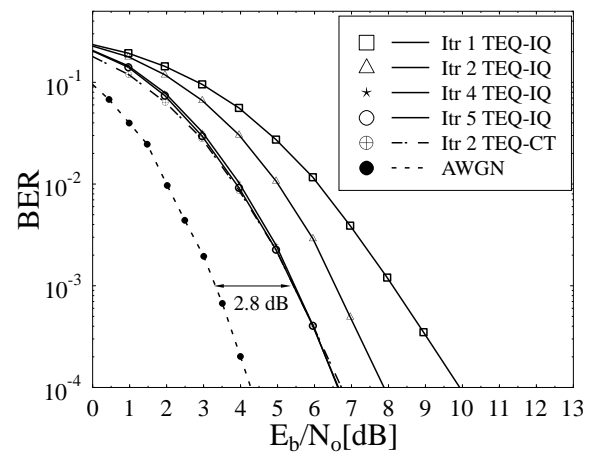

Figure 6: Performance of the TEQ-IQ using iterative CIR estimation and the TEQ-CT having perfect CIR information for a convolutional-coded 4-QAM system possessing a channel interleaving depth of 6912 bits over the equally-weighted, three-path Rayleigh fading CIR of Equation 4 using a normalised Doppler frequency of $3.3 \times 10^{-5}$ and burst-invariant fading.

\section{RESULTS AND DISCUSSION}

Our investigations suggested that after two turbo equalisation iterations the performance of the TEQ-CT having perfect CIR information did not improve significantly, despite invoking further iterations. We used the term critical number of iterations, in order to denote the number iterations, which was two in this case. When employing the TEQ-IQ receiver using iterative CIR estimation, the performance obtained after four and five turbo equalisation iterations was similar, as shown in Figure 6. Hence, the critical number of iterations performed by the TEQ-IQ receiver was four. The performance achieved by the TEQ-IQ using iterative CIR estimation after four iterations was also observed to be similar to that obtained by the TEQ-CT having perfect CIR information after two iterations in Figure 6. Using Equations 6 and 7, the complexity of TEQ-IQ was found to be a factor 1.25 lower than that of the TEQ-CT.

As a further set of results, Figure 7 displays the performance of the TEQ-CT receiver having perfect CIR information for 16-QAM transmitted over the Rayleigh fading channel of Equation 4. We found that the critical number of iterations was three, when employing the 16-QAM TEQ-CT receiver, while in Figure 7 the critical number of iterations was six, when employing the 16-QAM TEQ-IQ using iterative CIR estimation over the same channel. The performance achieved by the TEQ-IQ receiver after six iterations was observed to be similar to that obtained by the TEQ-CT receiver after three iterations in Figure 7. Furthermore, the complexity of the TEQ-IQ was found to be a factor of 7.17 lower than that of the TEQ-CT. Examining the performance of our 64-QAM system over the same dispersive 


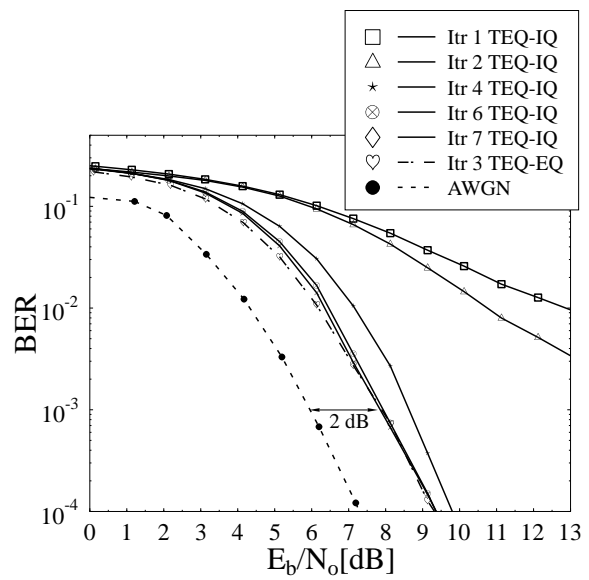

Figure 7: Performance of the TEQ-IQ using iterative CIR estimation and the TEQ-CT having perfect CIR information for a convolutional-coded 16-QAM system possessing a channel interleaving depth of 13824 bits over the equally-weighted three-path Rayleigh fading CIR of Equation 4 using a normalised Doppler frequency of $3.3 \times 10^{-5}$ and burst-invariant fading.

Rayleigh fading channel in Figure 8, it was observed that the critical number of iterations was ten. After six turbo equalisation iterations the performance of the TEQ-IQ using iterative CIR estimation at $\mathrm{BER}=10^{-3}$ was only $1.5 \mathrm{~dB}$ from the decoding performance curve over the non-dispersive Gaussian channel, as shown in Figure 8. Simulations could not be conducted for the 64-QAM TEQ-CT system, since the CT-EQ required $64^{2}=4096$ states and 64 transitions per state, hence it was too complex to be implemented.

\section{CONCLUSION}

It was observed in Figures 6 and 7, respectively, that the reduced complexity turbo equaliser, namely the TEQ-IQ scheme, employing two I/Q-EQs and iterative CIR estimation was capable of achieving the same performance as the significantly higher-complexity TEQCT scheme having perfect CIR information. Specifically, for 4-QAM and 16-QAM, a complexity reduction factor of 1.25 and 7.17 was obtained, respectively. For 64-QAM, we were unable to simulate the TEQ-CT due to the high number of trellis states required. However, for the TEQ-IQ receiver, the performance observed in Figure 8 at BER $=10^{-3}$ was only $1.5 \mathrm{~dB}$ from the best-case decoding performance derived from the nondispersive Gaussian channel.

\section{Acknowledgement}

This work has been funded in the framework of the IST project IST-1999-12070 TRUST, which is partly funded by the European Union. The authors would like to acknowledge the contributions of their colleagues.

\section{REFERENCES}

[1] C. Douillard, A. Picart, M. Jézéquel, P. Didier, C. Berrou, and A. Glavieux, "Iterative correction of intersymbol interference: Turbo-equalization," European Transactions on

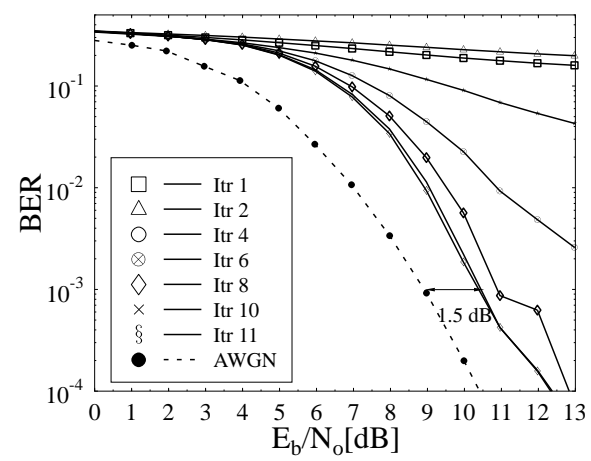

Figure 8: Performance of the TEQ-IQ using iterative CIR estimation for a convolutional-coded 64-QAM system possessing a channel interleaving depth of 20736 bits over the equally-weighted three-path Rayleigh fading CIR of Equation 4 using a normalised Doppler frequency of $3.3 \times 10^{-5}$ and burst-invariant fading.

Communications, vol. 6, pp. 507-511, September-October 1995.

[2] M. J. Gertsman and J. L. Lodge, "Symbol-by-symbol MAP demodulation of CPM and PSK signals on Rayleigh flatfading channels," IEEE Transactions on Communications, vol. 45, pp. 788-799, July 1997.

[3] A. Knickenberg, B. L. Yeap, J. Hàmorskỳ, M. Breiling, and L. Hanzo, "Non-iterative Joint Channel Equalisation and Channel Decoding," IEE Electronics Letters, vol. 35, pp. 1628-1630, 16 September 1999.

[4] A. Glavieux, C. Laot, and J. Labat, "Turbo Equalization over a frequency selective channel," in Proceedings of the International Symposium on Turbo Codes \& Related Topics, (Brest, France), pp. 96-102, 3-5 September 1997.

[5] M. S. Yee, B. L. Yeap, and L. Hanzo, "Iterative Radial Basis Function Assisted Turbo Equalisation," in Proceedings of the IEEE Vehicular Technology Conference 2000, (Tokyo, Japan), pp. 640-644, 15-18 May 2000.

[6] C. H. Wong, B. L. Yeap, and L. Hanzo, "Wideband burstby-burst adaptive modulation with turbo equalization and iterative channel estimation," in Proceedings of the IEEE Vehicular Technology Conference 2000, (Tokyo, Japan), pp. 2044-2048, 15-18 May 2000.

[7] A. Klein, R. Pirhonen, J. Sköld, and R. Suoranta, "FRAMES Multiple Access Mode1 - Wideband TDMA with and without spreading," in Proceedings of the IEEE International Symposium on Personal, Indoor and Mobile Radio Communications 1997, (Helsinki, Finland), pp. 37-41, 1-4 September 1997.

[8] L. Hanzo, W. Webb, and T. Keller, Single and Multicarrier Quadrature Amplitude Modulation. John-Wiley IEEE Press, 2000.

[9] P. Robertson, E. Villebrun, and P. Hoeher, "A Comparison of Optimal and Sub-Optimal MAP Decoding Algorithms Operating in the Log Domain," in Proceedings of the International Conference on Communications, (Seattle, United States), pp. 1009-1013, 18-22 June 1995.

[10] L. R. Bahl, J. Cocke, F. Jelinek, and J. Raviv, "Optimal Decoding of Linear Codes for Minimising Symbol Error Rate," IEEE Transactions on Information Theory, pp. 284-287, March 1974.

[11] S. Haykin, Adaptive Filter Theory. Prentice Hall, 1996. 